\title{
ON THE EXISTENCE OF THE GREEN'S FUNCTION FOR THE MOST GENERAL SIMPLY CONNECTED PLANE REGION
}

\author{
BY
}

W. F. OSGOOD

The problem of mapping the interior of a simply connected plane region $T$ conformally on the interior of a circle depends for its solution on the proof of the existence of the Green's function corresponding to $T$, i. e., of a function $u$ satisfying the following conditions :

(1) Except at a single arbitrarily chosen interior point $O$ of $T, u$ is single valued and harmonic throughout $T$. By harmonic is meant that $u$ satisfies Laplace's equation, $\Delta u=0$;

(2) At the point $O, u$ becomes discontinuous like $\log 1 r$, or

$$
u=\log 1 / r+u^{\prime},
$$

where $u^{\prime}$ is harmonic throughout the neighborhood of $O, r$ denoting the distance of the variable point $(x, y)$ from $O$;

(3) The boundary values of $u$ are all 0 , i. e., when the point $(x, y)$ approaches from the interior of $T$ an arbitrary point of the boundary of $T, u$ approaches the value 0 .

Hitherto the existence of the Green's function has been established $(\alpha)$ for regions $T$ bounded by a finite number of pieces of analytic curves (ScHWARZ), and $(b)$ for regions $T$ bounded by a finite number of pieces of regular curves (Paraf and Painlevé). By a regular curve is meant one that has a tangent that turns continuously as a variable point traces out the curve. Cf. PrCard, Traité, vol. II, chs. III, IV, X. In these cases it is shown furthermore that the boundary of $T$ is transformed continuously into the boundary of the circle. In the present paper it is proposed to establish the existence of the Green's function for the most general $\uparrow$ simply connected plane region $T$. Such regions include $(a)$ regions whose boundary is any Jordan curve $; \ddagger(\beta)$ regions whose boundary is more

* Presented to the Society June 29, 1900. Received for publication June 23, 1900.

$\dagger$ A single exceptional case is to be noted. If the image of $T$ on the sphere of stereographic projection consisted of the complete sphere less one point, the theorem would not hold.

$\ddagger$ By a Jordan curve is meant a curve of the general class of continuous curves without multiple points, considered by Jordan, Cours d'Analyse, vol. I, 2 d edition, 1893, p. 90. Cf. HurwITZ's geometric statemant of JORDAN's results, Zurich address, International Congress of Mathematicians, 1897. 
general. As an example of the latter class of regions consider the following. Assume on the axis of $x$ a set of points perfect, but not dense throughout any interval whatsoever.* Erect at each point of this set a perpendicular of unit length, drawn in the direction of the positive axis of $y$. The part of the upper half plane that remains after these lines have been cut out forms a region $T$ in point. If, in particular, the set of points on the axis of $x$ was so chosen that its content is positive, the content of the boundary of $T$, regarded as a set of points lying in a two-dimensional space, will likewise be positive. Moreover, the points of this boundary present a peculiarity not shared by the points of a Jordan curve. There are namely points in this set that must be regarded as points of the boundary of $T$, in as much as portions of $T$ lie in every neighborhood of these points ; and yet it is impossible to approach these points from the interior of $T$ along a continuous curve; that is to say, if $A$ denotes such a point and a circle of suitable radius $\epsilon$ is described with $A$ as center, then it is, of course, possible to join an arbitrary point, say $O$, of $T$ with a point of $T$ lying in the circle by means of a curve lying wholly within $T$. But if now a second circle of suitable radius $\epsilon^{\prime}$ is drawn about $A$, it will not be possible to continue the above path to a point in the inner circle without first passing out of the larger circle. In the above example, any interior point on a line about which lines cluster on both sides will serve as a point $A$.

The existence of points $A$ makes it necessary to define more precisely what form condition (3) in the definition of the Green's function shall take on. I will therefore state this condition as follows: If $p_{1}, p_{2}, \ldots$ are any set of points lying within $T$ and having as their only limiting point (Häufungsstelle) the point $A$; if, moreover, the value of $u$ in $p_{n}$ be denoted by $u_{p^{\prime \prime}}$, then

$$
\mathbf{I}_{n=\infty} u_{\mu_{n}}=0 \text {. }
$$

Proof of the Existence Theorem. $†$-We will first assume for the sake of simplicity that $T$ lies wholly in the finite region of the plane. Let the plane be divided into equal squares, $2^{-n}$ on a side, by means of the axes of $x$ and $y$ and lines drawn parallel to these axes. When $n$ is taken sufficiently large, some of these squares will lie wholly within $T$. Let $C$ be such a square and denote by $C_{n}$ the largest simply connected region that can be formed out of $C$ and other

\footnotetext{
* A suitable set for present purposes is one of which I have made frequent use; cf. A merican Jourual of Mathematics, vol. 19, 1897, p. 167 ; Cambridge Colloquium, Bulletin of the A merican Mathematical Society, ser. 2, vol. 5, 1898 : Lecture VI, p. 82.

† The method used in this proof is in substance the same as the one given by PoINCARÉ in his paper on the representation of an analytic configuration : $\quad u=f(z)$, by means of a pair of single valued functions of a parameter: $z=\phi(t), w=\psi(t)$; Bulletin de la Société mathématique de France, vol. 11, 1833, p. 112. Cf. Cambridge Colloquium, above cited, Lectures II, III.
} 
squares lying, like $C$, within $T$. When $n$ increases indefinitely, any preassigned point of $T$ finally will be included in $C_{n}$.

Denote by $u_{n}$ the Green's function for $C_{n}$ having $O$ as pole ( $n$ being taken large enough so that $O$ will be an interior point of $C_{n}$ ). Then $u_{n}$ is positive throughout $C_{n}$ and increases at each point of $C_{n}$ when $n$ increases. In fact, consider the function

$$
u_{n+1}-u_{n}
$$

throughout $C_{n}$. This function is harmonic at every interior point of $C_{n}$ and approaches continuous boundary values along the boundary of $C$, that are sometimes positive and never negative. Hence the function is positive throughout the whole interior of $C_{n}$. Furthermore, $u_{n}$ converges toward a limit, as will presently be shown, when $n$ becomes infinite, and thus by HarNack's theorem* the limit $u$ of $u_{n}$ :

$$
\mathbf{L}_{n=\infty} u_{n}=u,
$$

is a function that satisfies at least the first two conditions that the Green's function for $T$ must fulfill. We shall show that $u$ also satisfies the third condition and thus is the desired function. First, however, to show that $u_{n}$ actually approaches a limit. The proof for the special case we are consiclering (namely, where $T$ is of finite extent) can be given at once by considering, say, the Green's function $U$ for a circle with $O$ as center and large enough to contain the whole region $T$. Forthe difference

$$
U-u_{n}
$$

is positive throughout the whole extent of $C_{n}$. Thus $u_{n}$, always increasing with $n$, never exceeds $U$ and hence approaches a limit at a single point, say, of $C^{\gamma}$, so that HARNACK's theorem is now applicable.

This mode of proof is typical for the general case, but it is necessary to use a more complicated function $U$ to establish the convergence. Such a function we will now introduce, choosing it at once in such a way that it can be used to show that the limiting function $u$ satisfies condition (3) too.

Let $a, b, c$ be any three points of the plane of the complex variable $\approx$, and let the plane be cut from $a$ to $c$ along the arc $a b c$ of the circle passing through these three points. Then the plane, thus bounded, can be mapped conformally on a quadrilateral of a second plane (the $w$-plane), this quadrilateral being formed by a triangle whose sides are arcs of circles tangent to each other at the

* Harnack's theorem asserts that if a function $u_{n}$ is harmonic throughout a region $T$ for all values of $n \geqq m$ and if $u_{n}$ increases at all points of the region when $n$ increases ; if furthermore at one single interior point of $T u_{n}$ approaches a limit when $n$ becomes infinite, then $u_{n}$ converges at all interior points of $T$ and the limit $u$ is harmonic throughout the interior of $T$. Cf. HARNack, Logarithmixches l'otential, Leipzig, 1887, p. 167. 
vertices, and an adjacent triangle formed by reflecting the first triangle in one of its sides. Construct now by successive reflections the complete Riemann's surfaces that belong to the analytic function and its inverse, defined by the conformal transformation just considered. The result is the familiar modular figure* of the $w$-plane with the infinitely many-leaved surface of the z-plane having logarithmic branch points at $a, b, c$.

Now choose at pleasure a point $O$ in an arbitrary leaf of the $z$-surface distinct from the points $a, b, c$, and denote its image in the $w$-plane by $O^{\prime}$. Let $U^{\prime}$ be the Green's function for the fundamental circle of the $w$-figure, having its pole in $O^{\prime}$, and denote the same function transferred to the $\approx$-surface, by $U . U$ is then a function single-valued and harmonic on the $z$-surface except at the one point $O$, where it becomes logarithmically infinite ; and, moreover, $U$ approaches tice value 0 when the point $(x, y)$ approaches any one of the points $a, b, c$ along any path whatever, or, more generally, when $(x, y)$ passes through the points of a set $p_{1}, p_{2}, \cdots$, lying at pleasure on the surface, but having as the only point about which they cluster one of the points $a, b, c$.

Returning now to the region $T$, let us choose three points in the boundary of $T$ at pleasure and denote them by $a, b, c$. Form the function $U$ just defined for these three points and for the point $O$ already selected as the pole $O$ of $U$. Then $U$, regarded as a function restricted to the domain $T$, is single valued throughout this domain, becomes logarithmically infinite in $O$, and is positive along the whole boundary of any $C_{n}$. Hence

$$
u_{n}<U
$$

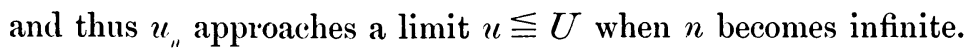

Finally, it can be shown by means of the function $U$ that $u$ approaches the boundary value 0 when the point $(x, y)$ approaches $a$, or more generally, when a set of points $p_{1}, p_{2}, \cdots$ is so taken in $T$ that $\lim p_{n}=a$, when $n=\infty$. For the corresponding values of $U: U_{\nu_{1}^{\prime}}, U_{\mu^{\prime}, 2}, \ldots$ approach 0 as their limit, and $0<u \leqq U$.

Thus the proof of the existence theorem is complete in ease $T$ lies wholly in the finite region of the plane, or what is in substance the same thing, in case there exist points of the plane external to $T$ and its boundary. If, however, $T$ and its boundary cover the complete sphere of stereographic projection, the proof. requires a slight modification in the construction of the regions $C_{n}$. It will no longer be feasible to associate with $C$ all the squares that lie in $T$ and form with $C$ a simply connected region, for this region might extend to infinity. It is sufficient, however, to confine oneself to those squares that lie wholly in $T$ ' and simultaneously in a circle with center $O$ and radius $n$.

\footnotetext{
* For an elementary discussion of the transformation and the functions thus defined cf. Cambridge Colloquium, above cited, Lecture I.
} 
The proof is now complete. It can readily be shown that the function $u$ thus determined is the only function satisfying the three conditions for the Green's function. Thus the most general transformation that carries the interior of a circle over conformally into itself, no assumption being made about the boundary, is a linear transformation; and hence the conformal transformation of the interior of an arbitrary simply connected region $T$ on the interior of a circle depends on three real parameters. These may be taken as the coördinates of the point $O_{1}$ of the circle into which $O$ is transformed and the angle that the image of a specified curve through $O$ makes with the radius of the circle drawn to $O_{1}$. It remains to investigate whether the boundary of $T$ (or a piece of it) is a Jordan curve, this boundary goes over continuously into the boundary of the circle.

The theorem of this paper justifies the conclusion that the function $\phi(t) \mathrm{em}$ ployed by Poincare* may be taken as an automorphic function and makes probable that the analytic configuration $w=f(z)$ may still be represented in a satisfactory manner by means of the parameter $t$ in the neighborhood of its singular points. Such a neighborhood will not, to be sure, be represented by the neighborhood of an interior point of the $t$-circle. But if it turns out, as I hope to show later, that it is represented by the neighborhood of a parabolic vertex, a desirable result will have been established.

ON the Atlantic,

June 11, 1900.

* loc. cit. 